\title{
Vector Median Filters Based on Noise Detection for Color Images
}

\author{
J. Wang \\ Department of Electronics \& Information Engineering \\ Ankang University \\ Ankang, China, 725000
}

\begin{abstract}
A novel filtering method is proposed in this paper in order to improve sharpness and detail reserve ability of color image filter. Start with analyzing the correlation between neighboring pixels and the correlation between each image channel, the image is then processed with a preprocessing procedure. Then we adopt one step singular correlation method to detect noise and the noise been detected is then complemented using the most relevant vector filtering. The simulation results show that this method we proposed can not only effectively detect the pepper and salt noise but also effective in reservation and restore of image edge and details. The PSNR and MSE value of the proposed method are significantly improved.
\end{abstract}

Keywords-vector median filters; color image processing; noise detection; most relevant vector filtering

\section{INTRODUCTION}

Nowadays, color image processing technique is widely adopted in many areas. The effective removal of noises brought into images, during the transmission stage for instance, plays a significant role in image quality enhancing. Pepper and salt noise is usually brought into image during transmission and acquisition stage. Though color image can be seen as a combination of grey images of the three channels, unlike grey images which can adopt median filter directly, the direct apply of median filtering could result in damage of image hue due to its ignorance of the correlation between each channel[1-2]. Morillas proposed the Vector Median Filter which solved this problem commendably by filtering the pixels of color image as a vector consist of elements in every channel [3]. Similarly, researchers adopt different vector distances and central weighted method to process the vector pixel, that is, use the minimal vector of the filter window to replace current pixel. However, this procedure tends to blur the image in [4-6]. To further protect image details while restrain noise, the switch vector filter and adaptive vector median filter are proposed in [7-8]. Belard inelli presented an improved vector median filter on the basis of SVF and A VMF in [9]. In order to gain better filtering result with possible lower amount of computation, it detects noise using the peer group filtering which is based on the peer group concept before processing the image using the improved adaptive vector median filter. Yet the accuracy decreas es greatly when the noise level rises. On this account, the method is inadequate when it comes to high quality demand of image processing.

Aiming at the shortcomings of existing Singular Correlation and weight method on image compensation, this paper proposed a color image filtering method based on One-
Step Singular Correlation and the Most Relevant Vector Filtering. The implementing steps are given and the efficiency is proved through serious of simulation.

\section{DESCRIPTION OFT HE PROBLEM}

\section{A. One-Step Singular Correlation and Compensation Method}

The One-Step Singular Correlation and compensation method consists in two parts: detection and compensation of the singular value. Assume that $X_{n}=\left\{x_{n}, n=1,2, \cdots, N_{X}\right\}$ is an observable stationary random sequence and divide it equally into $\mathrm{M}$ subsamples. NM is the length of local sequence. According to the zero-delay and one-step-delay correlation function, we can obtain the singular correlation detection equation as follow

$$
\tilde{\sigma}_{\Delta x(1)}^{2} \leq \sigma_{\Delta x(1)}^{2}
$$

Where

$$
\begin{aligned}
\tilde{\sigma}_{\Delta x(1)}^{2} & =\frac{1}{N_{M}} \sum_{n=0}^{N_{M}-1}(x(n)-x(n-1))^{2} \\
\sigma_{\Delta x(1)}^{2} & =\frac{1}{N_{X}} \sum_{n=0}^{N_{X}-1}(x(n)-x(n-1))^{2}
\end{aligned}
$$

Eq. 1 is only established under non-interruption condition. If there existed singular points, the variance difference between the singular point and its adjacent points would be great, therefore leads to $\tilde{\sigma}_{\Delta x(1)}^{2}>\sigma_{\Delta x(1)}^{2}$. So we can decide whether there are singular points.

The One-Step Singular Correlation and compensation method achieved great results in singular values' recognition and processing of random signal (or sequence) as well as in the detection of singular values in images. Nevertheless, the compensation method is acquired based on the correlation of one dimensional sequence. Because image pixels have correlation with its neighbouring points, the compensation method we introduced above is not suitable for the compensation of two dimensional grayscale image and three dimensional color image.

\section{B. Median Filtering and Weighted Method}

The main theory of the vector filtering method in literature [1] is the calculation of the cumulative distance of $x_{i}$ to other vectors in the filter window. ${ }^{x_{i}}$ is a vector in the filter window. 


$$
y_{k}=\sum_{j=1}^{N}\left\|x_{i}-x_{j}\right\|_{k}
$$

Where $y_{k}$ is the cumulative distance of ${ }^{x_{i}}$. The vector $x_{1}$ which obtains the smallest vector ascending sequence $\left\{y_{1}, y_{2}, \cdots, y_{N}\right\}$ is picked as the replacement of the central vector. The median filter and other developed weighted filtering, processing the image pixels indistinguishably, could result in undesirable errors therefore blur image details. The adaptive weighted filter proposed in literature [4] uses neighbour differential method to decide the effectiveness of filtered central points. The corresponding weight is given to different pixels and the filtering is achieved by reconstructing the pixels. The output value (compensation value) can be calculated by the function below.

$$
x_{i j}^{*}=\sum_{i, j \in W} \frac{\exp \left[-\left(\sum_{i, j=1}^{4} r_{i j}\right)^{2} /(2 \times 59)^{2}\right]}{\sum_{i, j \in W} \exp \left[-\left(\sum_{i, j=1}^{4} r_{i j}\right)^{2} /(2 \times 59)^{2}\right]^{i j}} x_{i}
$$

Where $r_{i j}$ is the absolute difference between the certain pixel in the filter window $\mathrm{W}$ and the others pixels. This method is significantly better than regular filtering. But the reconstructing is easy to damage the pixels. The constant 4 and 59 is set up by experiments experience and need to be modified according to different processing object. In other words, its robustness is low.

\section{THE PROPOSED METHOD}

To overcome the drawbacks we've mentioned, a One-Step Singular Correlation and compensation method is proposed in this paper. First we using One-Step Singular Correlation enabled fast detection of the singular interference. Then use the corresponding sub-windows of each channel as the compensation value window for the calculation to find the most correlated vector pixels as the compensation value.

\section{A. Noise Detection Method}

For the pixels of color images, once any element of the R, $\mathrm{G}, \mathrm{B}$ channel is decided contaminated by noise, then the pixel is decided as contaminated. A preprocessing step is added in order to reduce the influence of image details and edges on noise detection, that is, weakening image detail and edges to highlight the character of noise before noise detection. The specific progresses are as follows.

Assume that the image to be processed is an $H \times L \times K$ color image, where $H$ represents row number, $L$ is the column number, $K$ is the channel number. If $K=1$, the image is grayscale. If $K=3$, the image is chromatic. In each channel, choose a sub-window $W$ with proper size using $x_{(D+1) / 2}$ as the central pixel. $W \in H \times L$ and $D$ is the total number of pixels in $W$. If $(D+1) / 2$ were not integer, it should be rounded up to an integer. The absolute difference between $x_{(D+1) / 2}$ and its neighboring pixels can be calculated by

$$
x_{i j k}^{\prime}=\frac{1}{D} \sum_{m n \in W}\left|x_{(D+1) / 2}-x_{m n k}\right| ; i, j \in W, k=1,2,3
$$

Then the new matrix $\left[x_{i j k}^{\prime}\right]_{H \times L \times K}$ of size $H \times L \times K$ is obtained, of which $k$ represents the number of its belonging channel.

Think each layer of the matrix as a two dimensional matrix, then transfer this matrix to a one dimensional sequence $X_{n}$ by jointing its rows end to end. $X_{n}=\left\{x(n), n=1,2, \cdots, N_{X}=H \times L\right\}$. Then $X_{n}$ is divided into $N_{X}$ sub-sequence whose length is $N_{M}$ and meets the condition $N_{X}=N_{S} \times N_{M}$. After obtained the one-step difference variance of the sub-sequence and the main sequence respectively, we applied function (1) to conduct the singular value detection. The singular value we obtained is replaced temporarily by the overall mean value of the sequence so that the detection can progressing faster and more accurate. By mark the singular point with 1 and others with 0 , the noise signature matrix is finally obtained.

\section{B. The Most Correlative Vector Compensation Method}

Now that the signature matrix is obtained, we process pixels marked by 1 with weighted vector median compensation method, while pixels marked by 0 remain unchanged. The sub-window $W$ is used as the compensation window of the pixel marked with 1 which is also the central pixel of the sub-window. For a random pixel $x_{i j k}$ in $W$, calculate its Euclidean distance to other pixels in $W$. Considering the amount of computation, here we use the weighted absolute distance and then calculate the summation. Finally we sum up all the corresponding points of every layer.

$$
\begin{gathered}
S\left(x_{i j}\right)=\sum_{k \in K} \sum_{m, n \in W} w_{i j k}\left|x_{i j k}-x_{m n k}\right| ; i, j \in W, W \in H \times L \\
\text { Where } w_{i j k}=\exp \left[\left(\sum_{d=1} r_{i j k}^{(d)}\right)^{2} / 2 \mu^{2}\right], \\
r_{i j k}^{d} \in\left\{r_{i j k}=\left|x_{i j k}-x_{m n k}\right|, m, n \in W, k \in K\right\}, \\
r_{i j k}^{d} \leq r_{i j k}^{d+1}, d=1,2, \cdots D-1, u=\frac{1}{H \times L \times K} \sum_{k \in K} \sum_{m, n \in W} x_{m n k} .
\end{gathered}
$$

Therefore we can get a set $S\left(x_{i j}\right)$ formed by D summations and then find out the vector $x_{i j}$ which could obtain the smallest $S\left(x_{i j}\right)$.

$x_{i j}$ is used as the compensation $x^{*}$ to replace the corresponding pixel of the singular point we detected.

$$
x_{i j}^{*}=\left\{x_{i j} \mid \min \left\{S\left(x_{i j}\right), i, j \in W\right\}\right\}
$$

From function 6 we can define that the most correlative vector compensation method is to pick the corresponding pixel 
of the smallest value of the set $S\left(x_{i j}\right)$ in the sub-window $W$ as the compensation value. The characteristic of this method is: the sub-window with proper size and shape is choose based on the theory that the closer pixel has the greater correlation; weight $w_{i j k}$ is proportional to $r_{i j k}$ in order to highlight the correlation difference between pixels in $W$;for the $k t h$ layer, $\left\{r(d)_{i j k}\right\}$ is the ascending ordered result of $\left\{r_{i j k}\right\}$ and the summation time is $D / 2$ so as to reduce the influence of the absolute difference on weight value; the overall mean value is used as the weight value so preliminary experiments is avoided. Therefore the method is more pervasive. All the parameters in function (5) and (6) can change along with the feature of the image to be processed, thus the method has better fle xibility and practicality.

Clearly the proposed method is consists in two parts: noise detection and compensation. We adopted a preprocessing method before the noise detection. Then the noise detection is preformed to obtain the noise signature matrix, following by the compensation step. The compensation process is to think the pixels whose element is marked as the noise ele ment as the noise pixels and process it with the most correlative vector compensation method.

\section{THE EXPERIMENTAL RESULTS AND ANALYSIS}

The proposed method and the VMF and FPG-IA VMF is tested with picture "Lena" which is in $256 \times 256$ size and have less details and the picture "Hats" which is in $256 \times 256$ size and have more details. The noise images are the orig inal pictures contaminated by $10 \%$ and $30 \%$ pepper and salt noise respectively. Figs. 1-2 give the comparis on of filtering results of FPG-IA VMF and OSSC-MRVF. In terms of the vision effect of experimental results, regardless of the noise level, the processing effect of Fig. 1-2(d) is obviously better than Figs. 1-2(c). Background and edges of the Hats is more exquisite in Figs. 1-2 (d) therefore it is proved that the proposed method is better than the FPG-IA VMF method of sharpness. Figs. 1-2(d) is the most similar to the original image, indicates that the proposed method can retain and restore image edges and details better. Therefore it is proved that the proposed method is not only efficient in noise detection but also in compensation of the correlation.
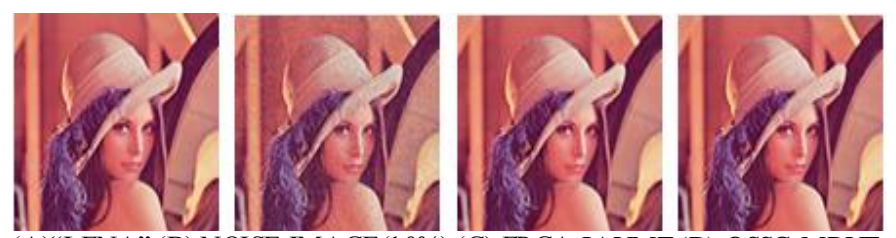

(A)“LENA" (B) NOISE IMAGE(10\%) (C) FPGA-IAVMF (D) OSSC-MRVF

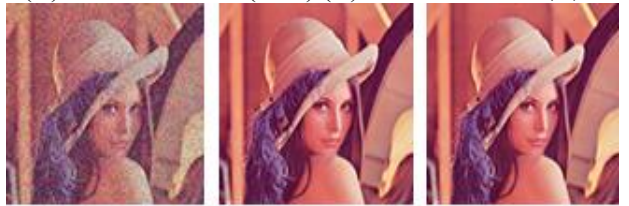

(E) NOISE IMAGE(30\%) (F) FPGA-IAVMF (G) OSSC-MRVF

FIGURE I. COMPARISON OF FILTERING RESULTS USING DIFFERENT APPROACHES FOR "LENA".
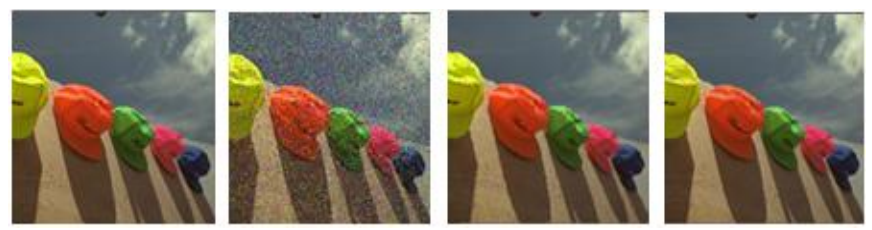

(A)“HAT” (B) NOISE IMAGE(10\%) (C) FPGA-IAVMF (D) OSSC-MRVF
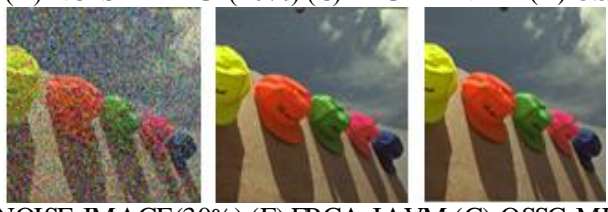

(E) NOISE IMAGE(30\%) (F) FPGA-IAVM (G) OSSC-MRVF

FIGURE II. COMPARISON OF FILTERING RESULT S USING DIFFERENT APPROACHES FOR "HAT".

We adopted MSE and PSNR as the objective assessing criterion. Table 1 shows the value of the criterion of the filtering results of each method. It is obvious that the proposed method has lower MSE than VMF and FPG-IA VMF. As the noise level increases, MSE of the proposed method remain relatively stable, indicates that the proposed method is more robust. Accordingly, the PSNR of the proposed method is higher than VMF and FPG-IA VMF and haven't changed much as the image complex rate rises, which demonstrates that the proposed method can restore the image preferably and is better in protecting image edges and details. Previous study and experiments shows that the proposed method has significantly improved performance in pepper and salt noise reduction and image edges and details protection both on the vision effect and the objective assessing criterion

TABLE I. ASSESSMENT DATA OF DIFFERENT METHODS.

\begin{tabular}{|c|c|c|c|c|c|c|c|}
\hline \multirow{2}{*}{$\begin{array}{l}\text { Metho } \\
\text { d }\end{array}$} & \multirow{2}{*}{$\begin{array}{c}\text { Asse } \\
\text { ssme } \\
\text { nt }\end{array}$} & \multicolumn{2}{|c|}{$10 \%$ noise } & \multicolumn{2}{|c|}{$20 \%$ noise } & \multicolumn{2}{|c|}{$30 \%$ noise } \\
\hline & & lena & hat & lena & hat & lena & hat \\
\hline \multirow[b]{2}{*}{ VMF } & MSE & $\begin{array}{c}15.34 \\
0\end{array}$ & 7.25 & $\begin{array}{c}43.48 \\
4\end{array}$ & 1.42 & $150.9^{7}$ & 61.32 \\
\hline & $\begin{array}{c}\text { PSN } \\
\text { R }\end{array}$ & $\begin{array}{c}36.74 \\
0\end{array}$ & $\begin{array}{c}35.28 \\
6\end{array}$ & $\begin{array}{c}31.80 \\
9\end{array}$ & $\begin{array}{c}31.01 \\
9\end{array}$ & $\begin{array}{c}26.346 \\
4\end{array}$ & $\begin{array}{c}26.053 \\
9\end{array}$ \\
\hline \multirow{2}{*}{$\begin{array}{c}\text { FPGA } \\
- \\
\text { IAVM } \\
\text { F } \\
\end{array}$} & MSE & $\begin{array}{c}11.76 \\
0\end{array}$ & $\begin{array}{c}16.76 \\
0\end{array}$ & $\begin{array}{c}15.94 \\
9\end{array}$ & $\begin{array}{c}21.91 \\
1\end{array}$ & $\begin{array}{c}20.704 \\
9\end{array}$ & $\begin{array}{c}27.760 \\
0\end{array}$ \\
\hline & $\begin{array}{c}\text { PSN } \\
\text { R }\end{array}$ & $\begin{array}{c}37.46 \\
1\end{array}$ & $\begin{array}{c}35.88 \\
7\end{array}$ & $\begin{array}{c}36.13 \\
9\end{array}$ & $\begin{array}{c}34.72 \\
4\end{array}$ & $\begin{array}{c}34.765 \\
2\end{array}$ & $\begin{array}{c}33.696 \\
6\end{array}$ \\
\hline \multirow{2}{*}{$\begin{array}{l}\text { OSSC- } \\
\text { MRVF }\end{array}$} & MSE & $\begin{array}{c}1.476 \\
3\end{array}$ & $\begin{array}{c}7.577 \\
8\end{array}$ & $\begin{array}{c}4.592 \\
6\end{array}$ & $\begin{array}{c}11.21 \\
0\end{array}$ & 5.6687 & $\begin{array}{c}14.486 \\
1\end{array}$ \\
\hline & $\begin{array}{c}\text { PSN } \\
\text { R }\end{array}$ & $\begin{array}{c}46.37 \\
5\end{array}$ & $\begin{array}{c}39.33 \\
5\end{array}$ & $\begin{array}{c}42.86 \\
5\end{array}$ & $\begin{array}{c}37.63 \\
4\end{array}$ & $\begin{array}{c}40.595 \\
9\end{array}$ & $\begin{array}{c}36.521 \\
2\end{array}$ \\
\hline
\end{tabular}

\section{CONCLUSIONS}

This paper adopted one-step singular correlation method in the study of the singular value detection and analyzed the limitation of the existed methods. The one-step singular correlation and most relevant vector filtering are then proposed. This method realized the fast and accurate recognition of the singular interference using one-step singular correlation method. Noise pixels is then marked and processed with the most relevant vector compensation. Parameters of the proposed method have high universality. Finally, through experiments and analyzing the criterion, it is demonstrated that the proposed method has high performance in pepper and salt noise reduction and image edges and details protection as the 
MSE of the processed image decreased significantly and mean while the PSNR increased remarkably.

\section{REFERENCES}

[1] Astola J., Haavisto P., Neuvo Y., Vector median filters: Proceedings of the IEEE, 78(4), pp. 678-689, 1990.

[2] Karakes D. G., Trahanias P. E., Generalized multichannel image filtering structures. IEEE Transactions on Image Processing, 6(7), pp. 1038-1045, 1997.

[3] Morillas S., Gregori V., Perisfajame G., Isolating impulsive noise pixels in color images by peer group techniques. Computer Vision and Image Underst anding, 110 (1), pp. 102-116. 2008.

[4] Lukac R., Adaptive vector median filtering: Pattern Recognition Letters, 24 (12), pp. 1889-1899, 2003.

[5] [5] Liu C., Zhang X. H., Shi B., Application of one-step singular correlation in random across probability modeling. IEEE International Conference on Electronics and Optoelectronics. Piscataway, 2, pp 422425, 2011.

[6] Verma O. P., Hanmandlu M., A novel approach for edge detection using ant colony optimization and fuzzy derivative technique: Proceedings of the 2009 IEEE Internat ional Advance Computing Conference, pp. 12061212. 2009,

[7] Serra J., Introduction to mathematical morphology: Computer Vision, Graphics and Image Processing, 35(1), pp. 283-305, 1986.

[8] A. Gernot, K. Mario, W. Lu. Singular value correlation functions for products of Wishart random matrices. Journal of Physics: A, Mathematical and Theoretical. 46 (27), pp. 275205, 2013.

[9] Belardinelli P., Jalava A., Gross J., et al., Optimal spatial filtering for brain oscillatory activity using the Relevance Vect or Machine. Cognitive Processing. 14, pp. 357-369, 2013. 